\title{
A mixed AC/DC model for railway power systems
}

\author{
J. Muñoz-Riesco, E. Pilo, A. Fernandez \& P. Cucala \\ Instituto de Investigación Tecnológica, \\ E.T.S. de Ingeniería Univ. Pont. Comillas de Madrid, Spain
}

\begin{abstract}
A method to study railway power supply systems with AC power distribution networks and DC power feeding is presented in this paper. The aim of this work is to obtain a DC model of the whole system in which AC networks are converted into equivalent $\mathrm{DC}$ ones.

Relationships between AC and DC quantities on both sides are set using the equations of multiple bridge rectifiers as described in the literature. Particular DC models for transformers and 3-phase lines are used to represent the corresponding voltage drops. Specific base quantities are used for AC and DC parts of the circuit in order to obtain a simplified circuit.

This model is used to solve a DC load flow problem. Real values of voltages, intensities and power flows can be obtained by using the proper base quantities. Additionally a correction has to be made to improve the power flow results calculated previously, due to pure resistive representation of transformers and AC lines, so a fine approximation is achieved.

Keywords: railways, $A C / D C$ power supply system, equivalent DC model.
\end{abstract}

\section{Introduction}

Railways' power systems usually have an AC division (zones close to distribution networks) and a DC one (zones close to trains). This feature makes those structures difficult to deal with when an electrical study is required to be practised. Homogenization of the electrical circuit to AC or DC helps it to be solved by means of a load flow problem.

Converting the whole circuit to AC provides exact results, but it involves a great amount of variables and calculations. 
Otherwise, transforming the configuration to DC does not offer so good conclusions (because of the AC part's reactance) but is easier and the calculus load is lower.

The proposal of this paper is to establish a method to solve railways' power systems with DC magnitudes minimizing the error.

\section{Railway's power system description}

Railway's power system is usually fed in DC power, generally in $3000 \mathrm{~V}$ or $1500 \mathrm{~V}$. Figure 1 shows the typical structure in this type of arrangements. Electricity supply is done from different points of the distribution network, which will be designated supply points. Connected to those points through several 3-phase lines are located the traction transformers, which are commonly made up of a transformation stage and a rectification stage, generally consisting of multiple 12-pulse bridge rectifiers, Kaller [2]. Those traction transformers cause a voltage drop between the positive and negative overhead contact lines.

Electricity can be delivered from several traction transformers simultaneously. Sometimes, depending on the separation between these elements, a parallel feeding is done.

Railways' electrical systems are topologically similar to railways' networks; thus it involves marked radial features, Profillidis [3]; except from urban centres.

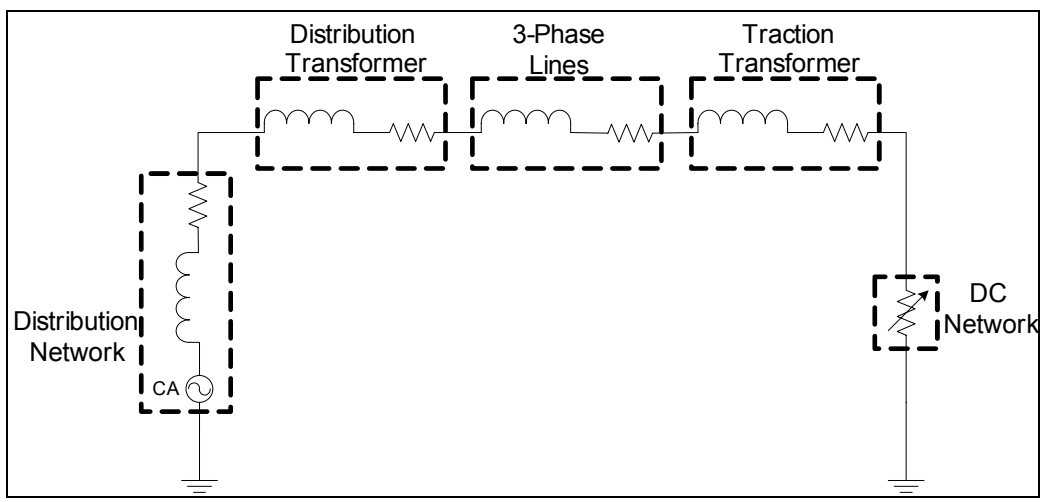

Figure 1: Railway's power system scheme.

\section{Electrical model}

As it has been observed previously, the AC part of the circuit will be adapted appropriately to come into being a single DC circuit. Figure 1 illustrates the group of elements, which compose the railway's power system.

The AC side can include several voltage levels: very high voltage (VHV) from the supply point to the distribution transformer (if it exists), high voltage (HV) in the 3-phase lines and medium voltage (MV) after the traction transformer. 
The DC part has only one voltage level, which depends directly on the medium voltage rectification given by the traction transformer. Every train will consume energy at this voltage.

The first step is to convert every quantity in the AC part to a unitary one that must be coherent with the values of power flow and voltage in the DC fraction of the circuit.

The second step is to convert the DC part to unitary quantities consistent with those used in the AC elements.

The third step is to adjust an equivalent resistance representative of resistance and reactance values in the $\mathrm{AC}$ section.

Power flow base DC quantities $\left(P_{B A S E} D C\right)$ and $\mathrm{AC}$ quantities $\left(\mathrm{P}_{\mathrm{BASE}} \mathrm{AC}\right.$ 3PHASE) are identical in the whole circuit, eqn. (1), for the reason that the transmitted power does not depend on the voltage or if it is AC or DC.

$$
P_{B A S E_{-} D C}=P_{B A S E_{-} A C_{-} 3-P H A S E}
$$

The relationship between traction transformer's low voltage side base AC quantities $\left(U_{B A S E_{-}-N_{-} A C-M V}\right)$ and $\mathrm{DC}$ base quantities $\left(U_{B A S E_{-} D C}\right)$, eqn. (3), is set by the rectification constant $\left(K_{R E C T}\right)$, eqn. (2), where $B$ is the number of rectifier's bridges in series ( 2 in this case), Kundur [1].

$$
\begin{gathered}
K_{R E C T}=\frac{3 \cdot \sqrt{6}}{\pi} \cdot B \\
U_{B A S E_{-} D C}=3 \cdot K_{R E C T} \cdot U_{B A S E_{-} L-N_{-} A C_{-} M V}
\end{gathered}
$$

Links between high base voltage $\left(U_{B A S E_{-}-N_{-} A C_{-} H V}\right)$ and AC base's medium voltage $\left(U_{B A S E} L_{-N} \_C_{-} M V\right)$ are fixed by transformation relationships of traction transformers ( $\left.K_{\text {TRAC-TRANSF }}\right)$, eqn. (5). Relationship between very high base voltage $\left(U_{B A S E} L-N_{A} A C V H V\right)$ and high $\mathrm{AC}$ voltage is set by distribution transformers $\left(K_{\text {DIST-TRANSF }}\right)$, eqn. $(\overline{7})$.

$$
\begin{aligned}
& \frac{U_{B A S E_{-} L-N_{-} A C \_M V}}{U_{B A S E_{-} L-N_{-} A C_{-} H V}}=K_{\text {TRAC-TRANSF }} \\
& U_{B A S E \_D C}=3 \cdot K_{R E C T} \cdot K_{\text {TRAC-TRANSF }} \cdot U_{\text {BASE_L-N_AC_HV }} \\
& \frac{U_{B A S E \_L-N_{-} A C_{-} H V}}{U_{B A S E_{-} L-N_{-} A C_{-} V H V}}=K_{\text {DIST-TRANSF }} \\
& U_{B A S E_{-} D C}=3 \cdot K_{\text {RECT }} \cdot K_{\text {TRAC-TRANSF }} \cdot K_{\text {DIST-TRANSF }} \cdot U_{\text {BASE_L-N_AC_VHV }}
\end{aligned}
$$

The specific treatment, description and modelling each device will receive is detailed following (Kaller [2], Hill [4, 5]).

\subsection{Distribution network}

It is modelled by its equivalent Thévenin dipole and it is connected to the supply point. The equivalent resistance $\left(R_{\text {DIST-NET }}\right)$ and reactance $\left(X_{\text {DIST-NET }}\right)$ models the voltage drop produced in the network because of trains' energy consumption.

The value of the voltage, reactance and resistance will be usually specified in total values so they must be converted to unitary quantities $\left(r_{\text {DIST-NET }}\right.$ and $x_{\text {DIST- }}$ $\left.{ }_{N E T}\right)$ of the $\mathrm{AC}$ zone, making use of base impedance $\left(Z_{B A S E_{-} A C_{-} V H V}\right)$, eqns. (9) and (10). 


$$
\begin{gathered}
Z_{B A S E_{-} A C_{-} V H V}=3 \cdot \frac{U_{B A S E_{-} L-N_{-} A C_{-} V H V}^{2}}{P_{B A S E_{-} A C_{-} 3-P H A S E}} \\
r_{D I S T-N E T_{-} B A S E_{-} A C}=\frac{R_{D_{I S T-N E T}}}{Z_{B A S E_{-} A C_{-} H V}} \\
x_{D I S T-N E T_{-} B A S E_{-} A C}=\frac{X_{D I S T-N E T}}{Z_{B A S E_{-} A C_{-} H V}}
\end{gathered}
$$

\subsection{Distribution transformer (optional)}

This transformer is used when traction transformer's voltage differs from the distribution network's one. The equivalent reactance and resistance is usually specified in unitary transformer values $\left(r_{\text {DIST-TRANSF }}\right.$ and $\left.x_{\text {DIST-TRANSF }}\right)$ and has to be converted to common unitary quantities ( $r_{\text {DIST-TRANSF_BASE_AC }}$ and $x_{\text {DIST-TRANSF_BASE_AC }}$ ), eqns. (13) and (14). Distribution transformer's high voltage base impedance $\left(Z_{N_{O} M_{-} A C_{-} H V_{-} D I S T-T R A N S F}\right)$ and high voltage zone's common base impedance $\left(Z_{B A S E A C H V}\right)$ are use to execute the conversion.

$$
\begin{aligned}
& Z_{N O M_{-} A C_{-} H V_{-} D I S T-T R A N S F}=3 \cdot \frac{U_{N O M_{-} L-N_{-} A C_{-} H V_{-} D I S T-T R A N S F}^{2}}{P_{N_{1} M_{-} A C_{-} 3-P H A S E_{-} D I S T-T R A N S F}} \\
& Z_{B A S E_{-} A C_{-} H V}=3 \cdot \frac{U_{B A S E_{-} L-N_{-} A C_{-} H V}^{2}}{P_{B A S E_{-} A C_{-} 3-P H A S E}} \\
& r_{\text {DIST-TRANSF } F_{-} B A S E_{-} A C}=r_{\text {DIST-TRANSF }} \cdot \frac{Z_{\text {NOM_AC_H _ } D I S T-T R A N S F}}{Z_{B A S E_{-} A C_{-} H V}} \\
& x_{\text {DIST-TRANSF_BASE_AC }}=x_{\text {DIST-TRANSF }} \cdot \frac{Z_{\text {NOM }_{-} A C_{-} H V_{-} D I S T-T R A N S F}}{Z_{B_{-} A S E_{-} A C_{-} H V}}
\end{aligned}
$$

\subsection{3-phase lines of connection between supply points and traction transformers}

These lines connect supply points (or distribution transformers if they exist) with traction transformers. The reactance and resistance will be normally arranged in total values ( $R_{3-P H A S E}$ LINES and $X_{3-P H A S E}$ LINES $)$ and will need to be converted to common unitary quantities ( $r_{3-P H A S E}$ LINES and $x_{3-P H A S E}$ LINES $)$ using high voltage zone's base impedance $\left(Z_{B A S E_{-} A C_{-} H V}\right)$, eqn. (16) and (17).

$$
\begin{aligned}
& Z_{B A S E_{-} A C_{-} H V}=3 \cdot \frac{U_{B A S E_{-} L-N_{-} A C_{-} H V}^{2}}{P_{B A S E_{-} A C_{-} 3-P H A S E}} \\
& x_{3-P H A S E-L I N E S_{-} B A S E_{-} A C}=\frac{X_{3-P H A S E-L I N E S}}{Z_{B A S E_{-} A C_{-} H V}} \\
& r_{3-P H A S E-L I N E S_{-} B A S E_{-} A C}=\frac{R_{3-P H A S E-L I N E S}}{Z_{\text {BASE_ } A C_{-} H V}}
\end{aligned}
$$




\subsection{Traction transformers}

Those elements provide medium voltage to the railways' electric power system. The equivalent reactance and resistance is known in unitary transformer values ( $r_{\text {TRAC-TRANSF }}$ and $\left.x_{\text {TRAC-TRANSF }}\right)$ so they have to be passed to common unitary

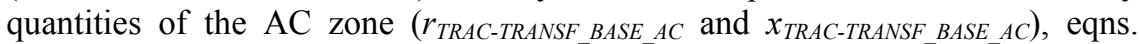
(20) and (21). Traction transformer's high voltage base impedance ( $Z_{N O M} A C$ HV TRAC-TRANSF $)$ and high voltage zone's common base impedance are use to carry out the adaptation.

$$
\begin{aligned}
& Z_{N O M_{\_} A C_{-} H V_{-} T R A C-T R A N S F}=3 \cdot \frac{U_{\text {NOM_L } L N_{-} A C_{-} H V_{-} T R A C-T R A N S F}^{2}}{P_{\text {NOM_A } A C_{-} 3-P H A S E_{-} T R A C-T R A N S F}} \\
& Z_{B A S E_{-} A C_{-} H V}=3 \cdot \frac{U_{B A S E_{-} L-N_{-} A C_{-} H V}^{2}}{P_{B A S E_{-} A C_{-} 3-P H A S E}}
\end{aligned}
$$

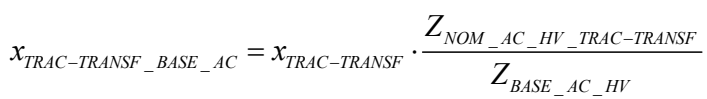

$$
\begin{aligned}
& r_{\text {TRAC-TRANSF }_{-} B A S E_{-} A C}=r_{\text {TRAC-TRANSF }} \cdot \frac{Z_{\text {NOM_AC_H }_{-} T R A C-T R A N S F}}{Z_{B_{1} A S E_{-} A C_{-} H V}}
\end{aligned}
$$

\subsection{DC network}

It is composed by the railway's overhead contact line, which transports the electric energy and are purely resistive; and by the train, moving charges that demand electrical power. The power base quantity will be identical to the $\mathrm{AC}$ one, eqn. (1), and AC low voltage and DC voltage are associated through the relationship of the rectifier, eqn. (3). Resistance of DC section $\left(R_{\text {LINES }}\right)$ of the circuit have to be transformed to DC unitary quantities $\left(r_{\text {LINES BASE DC }}\right)$ using the base impedance of this part of the circuit $\left(Z_{B A S E_{-} D C}\right)$, eqn. (23).

$$
\begin{aligned}
& Z_{B^{\prime} A E_{-} D C}=\frac{U_{B_{A S E_{-}} D C}^{2}}{P_{B_{A S E_{-} D C}}} \\
& r_{\text {LINES } B \text { BASE }_{-} D C}=\frac{R_{\text {LINES }}}{Z_{B A S E_{-} D C}}
\end{aligned}
$$

\subsection{Equivalent DC resistance}

The objective of solving a single DC circuit requires using an equivalent DC resistance $\left(R_{e q}\right)$ to obtain the real voltage and intensity current. In the $\mathrm{AC}$ zone, impedance consists in reactance, which causes voltage drop but not power lose; and in resistance, which causes voltage drop and also power loses. When a model is developed to emulate this electrical performance, it must origin both voltage drop (due to the reactance and resistance combination) and power loses (due to resistance). The method used to complete an expression that complies with the conditions given before will be exposed at the next point providing the equivalent resistance shown in eqn. (35).

The resultant circuit can be solved with a power flow, and because of DC features, angles will not have to be work out, simplifying the resolution. 


\subsection{Total quantities}

After the circuit resolution, every transformation can be undone; so voltages, currents and power flows are expressed in their real values.

\subsection{Power losses correction}

The results achieved with this method are quite acute, but if a better precision is needed for power losses, a correction can be made.

The electrical analysis of this adjustment is done in the following point, being eqn. (47) the expression that may correct the power losses.

\section{Electrical analysis}

Figure 2 matches up to an electrical module and angle study. Voltage applied to the system at the supply point is $V i$. The power transmission system is composed of transformers, 3-phase AC lines, and DC lines; and all together could be represented as an impedance (provided the quantities have a common base), designated $Z$ as $I$ is shown in eqn. (24). Impedance will have resistance $(R)$ due to transformers and DC lines and reactance $(X)$ because of transformers and AC lines, eqns. (25) and (26) respectively.

$$
\begin{aligned}
& \bar{Z}=R+j X \\
& R=R_{{\text {DIST }-N E T_{-} B A S E_{-} A C}}+R_{\text {DIST-TRANSF }_{-} B A S E_{-} A C}+ \\
& +R_{3-P H A S E-L I N E S_{-} B A S E_{-} A C}+R_{\text {TRAC-TRANSF }_{-} B A S E_{-} A C} R_{\text {LINES }_{-} B A S E_{-} D C} \\
& X=X_{{\text {DIST }-N E T_{-} B A S E_{-} A C}}+X_{{\text {DIST-TRANSF_- } B A S E_{-} A C}}+ \\
& +X_{3-P H A S E-L I N E S_{-} B A S E_{-} A C}+X_{\text {TRAC-TRANSF_BSE } B A S E_{-} A C}
\end{aligned}
$$

Trains are exposed to voltage $V o$ demanding an intensity current $I$. Vo must have the same angle as $I$ because the charge feature of them is purely resistive. On the other hand, $V i$ will have a different angle value produced by the reactance effect.

The method used simply tries to get the equivalent resistance value which makes $V o$ in the DC model to be as similar as possible as it is in the AC one. The principal hypothesis is to suppose that the angle between $V i$ and $V o$ is very small so the Taylor series can be used instead of trigonometric functions.

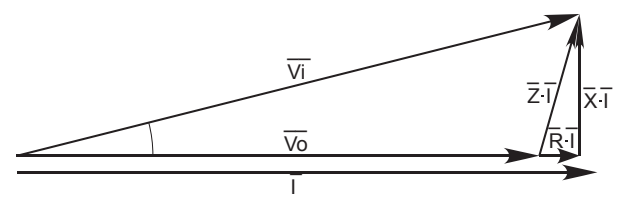

Figure 2: Phasorial diagram of relevant magnitudes in the real case. 
Substituting Taylor series into trigonometric functions, eqns. (27), (28) and (29); an expression for $\cos (\varphi)$ can be accomplished, eqn. (30).

$$
\begin{gathered}
\sin (\varphi)=\frac{X \cdot I}{V_{i}} \\
\varphi \approx \frac{X \cdot I}{V_{i}} \\
\cos (\varphi) \approx 1-\frac{1}{2} \cdot \varphi^{2} \\
\cos (\varphi) \approx 1-\frac{1}{2} \cdot\left(\frac{X \cdot I}{V_{i}}\right)^{2}
\end{gathered}
$$

Making use of the previous approximation, eqn. (30), the equivalent resistance, eqn. (34), will be calculated as the division of the voltage drop by the current intensity.

$$
\begin{gathered}
\Delta V=V_{i}-V_{o} \\
V_{o}=V_{i} \cdot \cos (\varphi)-R \cdot I \approx V_{i}-\frac{1}{2} \cdot \frac{(X \cdot I)^{2}}{V_{i}}-R \cdot I \\
\Delta V \approx \frac{1}{2} \cdot \frac{(X \cdot I)^{2}}{V_{i}}+R \cdot I \\
R_{e q}=\frac{\Delta V}{I} \approx \frac{1}{2} \cdot X^{2} \frac{I}{V_{i}}+R
\end{gathered}
$$

Finally, the approximation is settled as a function of the nominal demanded power through the respective element and the input voltage, eqn. (35).

$$
R_{e q} \approx \frac{1}{2} \cdot X^{2} \frac{P}{V_{i}^{2}}+R
$$

The result after the conversion in DC magnitudes is shown in Figure 3.

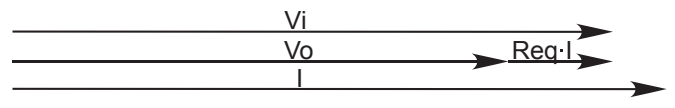

Figure 3: Phasorial diagram of relevant magnitudes in the simplified case.

This result depends on the demand of power, but the estimation has to be standardized to be applied in a general way for every component in the AC part. A power value must be introduced into the equation and it should be the real one demanded by the trains from this supply point, but it also should be constant. Because of that, a study to know which electric power grants better approximations needs to be done.

Below the real performance and the model are compared to identify the errors made because of approximations. Nominal power introduced in the equivalent resistance eqn. (35), and demanded power are the parameters that will determine the magnitude of errors. An example is proposed to establish comparisons between both models. It will receive typical values of input voltage, eqn. (36); resistance, eqn. (37); reactance, eqn. (38); and a grid of nominal power, eqn. (39), and demanded power, eqn. (40). 


$$
\begin{gathered}
V_{i}=1 p u \\
R=0.05 p u \\
X=0.15 p u \\
P_{\text {NOM }}=[0.25,0.50,0.75,1.00,1.25,1.50] p u \\
P_{D E M}=[0.25,0.50,0.75,1.00,1.25,1.50] p u
\end{gathered}
$$

Equation (41) is used to solve the real case, Figure 2. Equation (42) will be also employed with equation (42) to explain the simplified model's outcome, Figure 3. In both cases power consumption, eqn. (43), will be known and its value will affect the conclusions clearly.

$$
\begin{gathered}
V_{i}^{2}=\left(V_{o}+R \cdot I\right)^{2}+(X \cdot I)^{2} \\
V_{i}=V_{o}+R_{e q} \cdot I \\
P_{D E M}=V_{o} \cdot I
\end{gathered}
$$

Once both models have been worked out for the variation of parameters nominal power and demanded power, eqn. (39) and (40); voltage output, current and power losses are contrasted.

Relative errors $(\varepsilon)$ are obtained by means of eqn. (44) and applied to variables $V o, I$ and $L$ (power losses).

$$
\varepsilon_{P_{\text {NOM }}, P_{D E M}}=\frac{\operatorname{abs}\left(\text { Value }\left._{P_{\text {NOM }}, P_{\text {DEU }}}\right|_{\text {REAL }}-\text {-Value }\left._{P_{\text {NOM }}, P_{D E U}}\right|_{\text {MODEL }}\right)}{\text { Value } \left._{P_{\text {NOM }}, P_{D E M}}\right)}
$$

Mean errors $(\bar{\varepsilon})$ for each nominal power value is also useful to rate the model's goodness of fit. It is achieved using the probability each power consumption value has, eqn. (46).

$$
\begin{gathered}
\operatorname{Prob}_{\text {POWER }}=[0.30,0.20,0.30,0.15,0.10,0.05] \\
\bar{\varepsilon}_{P_{N O M}, P_{D E M}}=\sum_{i=P_{D E M}} \varepsilon_{P_{N O M}, i} \cdot \operatorname{Prob}_{i}
\end{gathered}
$$

Table 1 illustrates relative errors between real solution and model's solution for $V o$ module. Table 2 shows mean errors for each nominal power value.

Table 1: Relative error between real and model's Vo module value.

\begin{tabular}{|c|c|c|c|c|c|c|c|c|}
\cline { 3 - 9 } \multicolumn{2}{c|}{} & \multicolumn{9}{c|}{$\mathrm{P}_{\text {NOM }}[\mathrm{pu}]$} \\
\cline { 3 - 9 } \multicolumn{2}{c|}{} & 0.25 & 0.50 & 0.75 & 1.00 & 1.25 & 1.50 & 0.25 \\
\hline \multirow{4}{*}{\begin{tabular}{c}
$\mathrm{P}_{\text {DEM }}[\mathrm{pu}]$ \\
\cline { 2 - 9 }
\end{tabular}} & 0.25 & 0.0020917 & 0.074215 & 0.15064 & 0.22719 & 0.30386 & 0.38065 & 0.0020917 \\
\cline { 2 - 9 } & 0.70 & 0.18798 & 0.020522 & 0.14754 & 0.3162 & 0.48547 & 0.65536 & 0.18798 \\
\cline { 2 - 9 } & 1.00 & 1.5576 & 1.1343 & 0.70685 & 0.27516 & 0.16092 & 0.60172 & 1.5576 \\
\cline { 2 - 9 } & 1.25 & 3.3017 & 2.6832 & 2.0554 & 1.418 & 0.77029 & 0.11194 & 3.3017 \\
\cline { 2 - 9 } & 1.50 & 7.0122 & 6.101 & 5.1686 & 4.2134 & 3.2336 & 2.2273 & 7.0122 \\
\hline
\end{tabular}

Table 2: Mean error between real and model's Vo module value.

\begin{tabular}{|c|c|c|c|c|c|c|}
\hline $\mathrm{P}_{\mathrm{NOM}}[\mathrm{pu}]$ & 0.25 & 0.50 & 0.75 & 1.00 & 1.25 & 1.50 \\
\hline $\bar{\varepsilon}[\%]$ & 1.1472 & 0.88054 & 0.67096 & 0.58382 & 0.59525 & 0.68826 \\
\hline
\end{tabular}


Table 3 illustrates relative errors between real solution and model's solution for $I$ module. Table 4 shows mean errors for each nominal power value.

Table 3: Relative error between real and model's I module value.

\begin{tabular}{|c|c|c|c|c|c|c|c|c|}
\cline { 3 - 9 } \multicolumn{2}{c|}{} & \multicolumn{9}{c|}{$\mathrm{P}_{\text {NOM }}[\mathrm{pu}]$} \\
\cline { 3 - 9 } \multicolumn{2}{c|}{} & 0.25 & 0.50 & 0.75 & 1.00 & 1.25 & 1.50 & 0.25 \\
\hline \multirow{4}{*}{$\begin{array}{c}\mathrm{P}_{\text {DEM }} \\
{[\mathrm{pu}]}\end{array}$} & 0.25 & 0.0020916 & 0.07427 & 0.15087 & 0.22771 & 0.30478 & 0.3821 & 0.0020916 \\
\cline { 2 - 9 } & 0.50 & 0.18763 & 0.020518 & 0.14775 & 0.3172 & 0.48784 & 0.65969 & 0.18763 \\
\cline { 2 - 9 } & 0.75 & 0.64432 & 0.36752 & 0.087441 & 0.19599 & 0.48284 & 0.7732 & 0.64432 \\
\cline { 2 - 9 } & 1.00 & 1.5337 & 1.1215 & 0.70188 & 0.2744 & 0.16118 & 0.60536 & 1.5337 \\
\cline { 2 - 9 } & 1.25 & 3.1961 & 2.6131 & 2.014 & 1.3981 & 0.76441 & 0.11182 & 3.1961 \\
\cline { 2 - 9 } & 1.50 & 6.5527 & 5.7502 & 4.9146 & 4.043 & 3.1323 & 2.1787 & 6.5527 \\
\hline
\end{tabular}

Table 4: Mean error between real and model's I module value.

\begin{tabular}{|c|c|c|c|c|c|c|}
\hline $\mathrm{P}_{\text {NOM }}[\mathrm{pu}]$ & 0.25 & 0.50 & 0.75 & 1.00 & 1.25 & 1.50 \\
\hline $\bar{\varepsilon}[\%]$ & 1.1087 & 0.85369 & 0.65346 & 0.57367 & 0.59109 & 0.68945 \\
\hline
\end{tabular}

Table 5 illustrates relative errors between real solution and model's solution for Power losses. Table 6 shows mean errors for each nominal power value.

Table 5: Relative error between real and model's Power losses value.

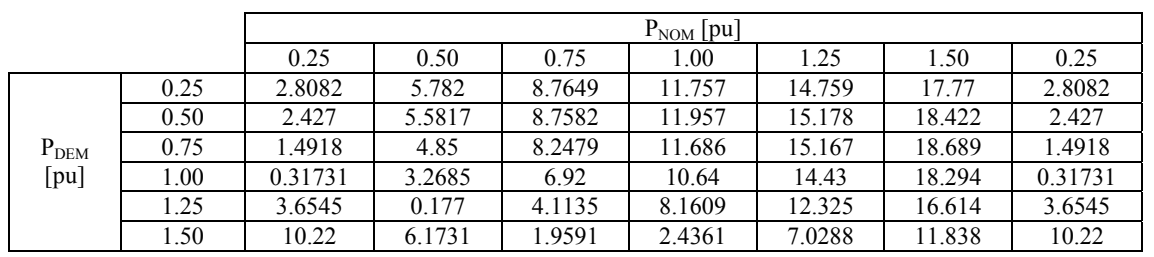

Table 6: Mean error between real and model's Power losses value.

\begin{tabular}{|c|c|c|c|c|c|c|}
\hline $\mathrm{P}_{\mathrm{NOM}}[\mathrm{pu}]$ & 0.25 & 0.50 & 0.75 & 1.00 & 1.25 & 1.50 \\
\hline $\bar{\varepsilon}[\%]$ & 2.6995 & 5.1225 & 8.4028 & 11.958 & 15.762 & 19.62 \\
\hline
\end{tabular}

Because of errors in estimation of power losses is not as good as it would be desirable, a correction is applied to improve the adjustment, eqn. (47).

$$
L_{\text {CORRECTED_MODEL }}=L_{M O D E L}-\left(R_{e q}-R\right) \cdot I^{2}
$$

Table 7 illustrates relative errors between real solution and model's solution for corrected Power losses. Table 8 shows mean error for each nominal power value.

Table 7: Relative error between real and corrected model's Power losses value.

\begin{tabular}{|c|c|c|c|c|c|c|c|c|}
\cline { 3 - 9 } \multicolumn{2}{c|}{} & \multicolumn{9}{c|}{$\mathrm{P}_{\text {NOM }}[\mathrm{pu}]$} \\
\cline { 3 - 9 } \multicolumn{2}{c|}{} & 0.25 & 0.50 & 0.75 & 1.00 & 1.25 & 1.50 & 0.25 \\
\hline \multirow{4}{*}{$\begin{array}{c}\mathrm{P}_{\text {DEM }} \\
{[\mathrm{pu}]}\end{array}$} & 0.25 & 0.0041832 & 0.14859 & 0.30196 & 0.45593 & 0.61049 & 0.76566 & 0.0041832 \\
\cline { 2 - 9 } & 0.50 & 0.37491 & 0.041036 & 0.29572 & 0.6354 & 0.97805 & 1.3237 & 0.37491 \\
\cline { 2 - 9 } & 0.75 & 1.2845 & 0.73375 & 0.17488 & 0.39226 & 0.96789 & 1.5522 & 1.2845 \\
\cline { 2 - 9 } & 1.00 & 3.0442 & 2.231 & 1.3995 & 0.54884 & 0.32163 & 1.2144 & 3.0442 \\
\cline { 2 - 9 } & 1.25 & 6.2901 & 5.1579 & 3.9875 & 2.7767 & 1.523 & 0.22351 & 6.2901 \\
\cline { 2 - 9 } & 1.50 & 12.676 & 11.17 & 9.5876 & 7.9226 & 6.1665 & 4.31 & 12.676 \\
\hline
\end{tabular}


Table 8: Mean error between real and corrected model's Power losses value.

\begin{tabular}{|c|c|c|c|c|c|c|}
\hline $\mathrm{P}_{\text {NOM }}[\mathrm{pu}]$ & 0.25 & 0.50 & 0.75 & 1.00 & 1.25 & 1.50 \\
\hline $\bar{\varepsilon}[\%]$ & 2.181 & 1.6818 & 1.2903 & 1.1377 & 1.178 & 1.3801 \\
\hline
\end{tabular}

Information given in Table 1, Table 3, Table 5 and Table 7 is illustrated graphically in Figure 4.

\section{Conclusion}

This model is being used in real cases to calculate the electrical power supply for the Spanish railway's power system. The achieved results, making use of the correct nominal power for each element, provide a fine approximation to the real solution.

Working with systems that combine AC and DC parts is facilitated by the exposed method, reaching a DC equivalent circuit that can be solved or studied simply.

As it is exposed in Figure 4, the best approximation is achieved when $P_{\text {NOM }}=1[\mathrm{pu}]$.

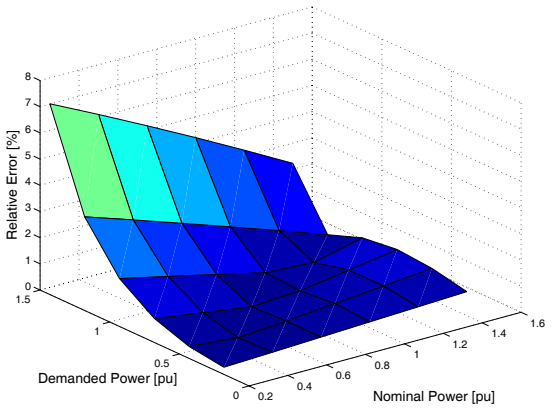

POWER LOSSES RELATIVE ERROR

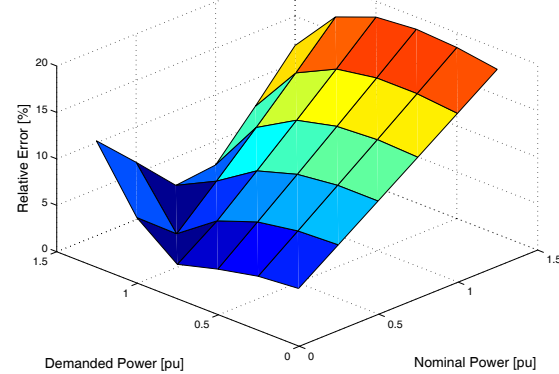

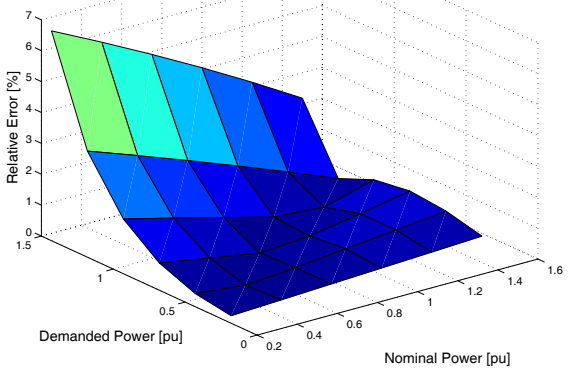

CORRECTED POWER LOSSES RELATIVE ERROR

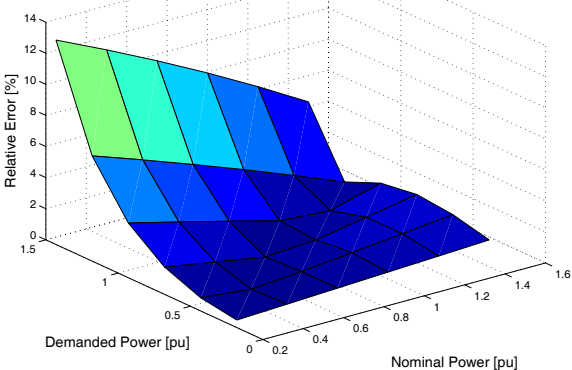

Figure 4: Relative error between real and corrected model's variables. 


\section{References}

[1] Kundur, P., Power system stability and control, McGraw-Hill, pp. 468-497, 1993.

[2] Kaller R.,Allenbach J.M., Traction électrique, Presses polytechniques et universitaires romandes: Lausanne, 1995.

[3] Profillidis V. A., Railway Engineering, Ashgate, 2000.

[4] Hill R. J., Brillante S., Souza C. R. de et al., Electrical material data for railway track transmission line parameter studies, IEE Proceedings on Electrical Power Applications 146 (No 1, January 1999): 60-68.

[5] Hill R. J., Carpenter D. C., Determination of rail internal impedance for electric railway traction system simulation, IEE Proceedings B 138 (No 6, November 1991): 311-321. 